\title{
Uncovering human mixed lineage kinase domain-like activation in necroptosis
}

\author{
Cristina D Guibao', Katherine Petrinjak \& Tudor Moldoveanu ${ }^{*, 1}$ \\ ${ }^{1}$ Departments of Structural Biology \& Chemical Biology \& Therapeutics, St. Jude Children's Research Hospital, Memphis, TN \\ 38105, USA \\ *Author for correspondence: tudor.moldoveanu@stjude.org
}

MLKL and its obligate upstream receptor interacting protein kinase 3 are essential components of necroptosis. It is well established that MLKL is the executioner of plasma membrane rupture in necroptosis. In healthy cells MLKL is dormant. Several dormant configurations have emerged from high-resolution structural studies revealing distinct mechanisms of MLKL autoinhibition in mammals. MLKL is activated through the concerted actions of receptor interacting protein kinase 3 , which phosphorylates MLKL, and, in the case of the human pathway, inositol phosphate (IP) metabolites synthesized by the IP kinases of the IP metabolic pathway. Here, we highlight recent progress toward understanding the mechanisms of regulation of human MLKL, and survey the latest opportunities for targeting MLKL in pathophysiology.

\section{Graphical abstract:}

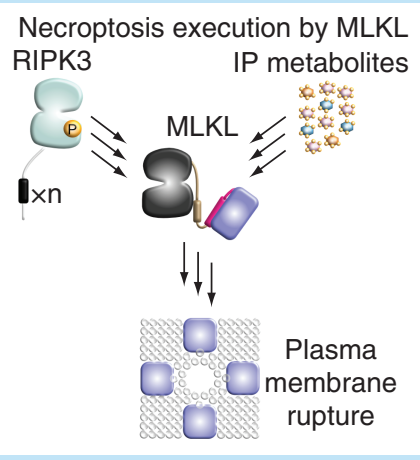

First draft submitted: 5 August 2019; Accepted for publication: 10 September 2019; Published online: 12 November 2019

Keywords: chemical probes $\bullet$ mixed lineage kinase domain-like $(\mathrm{MLKL}) \bullet$ necroptosis $\bullet$ oligomerization $\bullet$ phosphorylation $\bullet$ plasma membrane rupture $\bullet$ programmed cell death $\bullet$ pseudokinase $\bullet$ receptor interacting protein kinase 3 (RIPK3) • structural biology

\section{Necroptosis: a ubiquitous immunogenic cell death program in human pathophysiology}

Several sophisticated programmed cell death pathways have evolved in humans to cope with cellular stress [1]. Two of these pathways, apoptosis and necroptosis, are marshaled in pathophysiology to protect the integrity and homeostasis of tissues and organs through the removal of damaged, deleterious cells, including tumor and pathogeninfected cells. Necroptosis responds to stress by triggering the activation of various endogenous sensors that are an integral part of the innate immune response. Irrespective of triggering sensors, necroptosis involves the activation of a kinase, RIPK3 [2-4] and its obligate pseudokinase substrate, MLKL [5,6]. MLKL is the executioner of the plasma membrane rupture in necroptosis and the subject of this commentary. Because of its coarse nature, with dying cells releasing their contents into the surrounding environment, necroptosis is immunogenic. It is thought to elicit local and systemic innate and adaptive immune responses contributing significantly to health and disease [7-9]. 


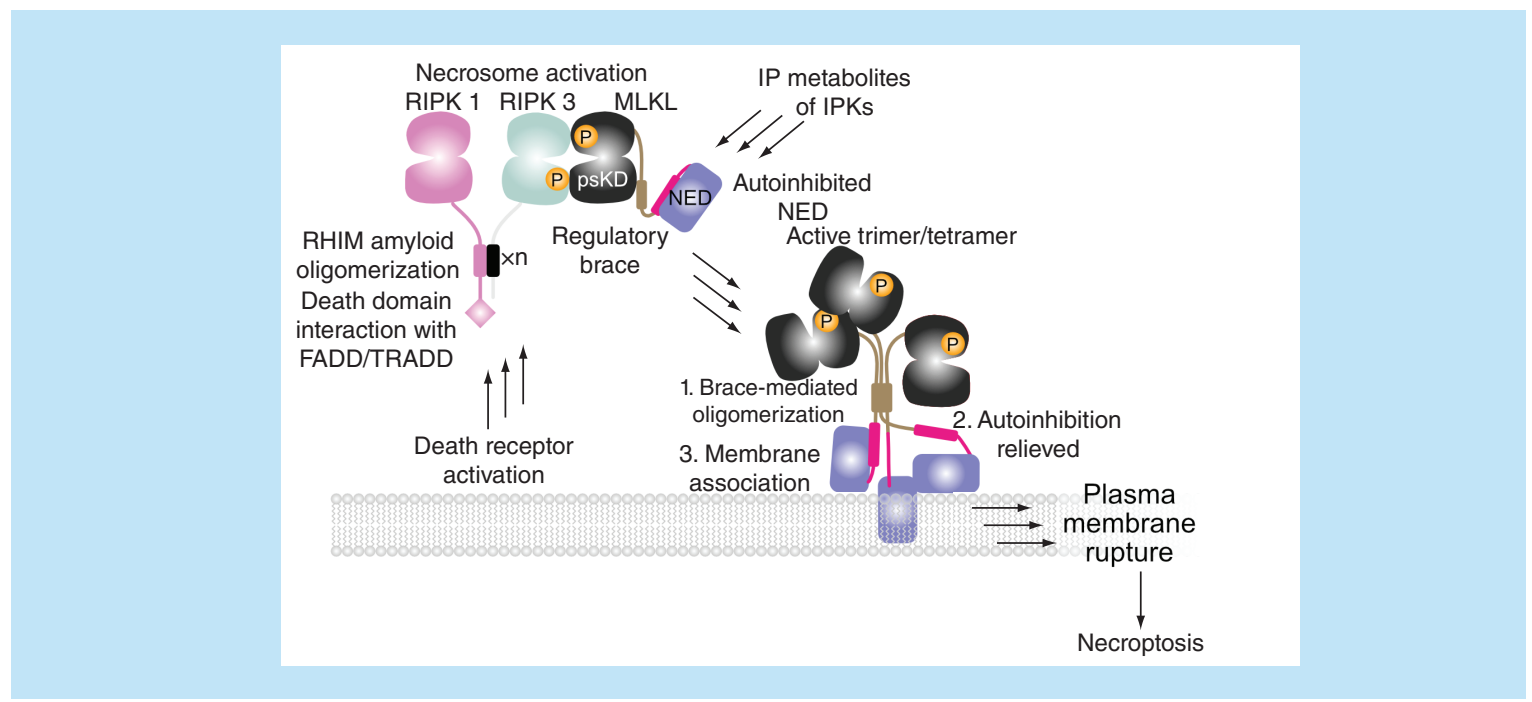

Figure 1. Necroptotic signaling activates mixed lineage kinase domain-like through receptor interacting protein kinase 3. Necroptosis signaling triggers assembly and activation of the necrosome complex formed by receptor interacting protein kinase 3 (RIPK3) and mixed lineage kinase domain-like (MLKL). The most common, canonical necrosome activation pathway involves death receptor signaling, which recruits and activates RIPK1. Under conditions of compromised caspase-8 activity, RIPK1 dissociates from the death receptor as part of a complex involving Fas-associated protein with death domain (FADD) and sometimes including Tumor necrosis factor receptor-associated death domain (TRADD). RIPK1 recruits RIPK3 to these complexes through their receptor interacting protein (RIP) homotypic interaction motifs, which promote amyloid oligomerization of these kinases. Two other RIP homotypic interaction motif-containing proteins, z-DNA-binding protein 1 (ZBP1)/ DNA-dependent activator (DAI) and Toll/IL-1 receptor domain containing adaptor-inducing interferon- $\beta$ (TRIF)/ Toll/interleukin receptor domain-containing adaptor molecule 1 (TICAM1), have been implicated in noncanonical RIPK3 activation (not shown), presumably also by promoting RIPK3 oligomerization. RIPK3 oligomers autoactivate through phosphorylation and possibly kinase domain dimerization. Active phospho-RIPK3 in turn phosphorylates MLKL pseudokinase domain (psKD) promoting its activation. Inositol phosphate (IP) kinases (IPKs) are essential in human MLKL (hMLKL) activation. They produce IP metabolites, which bind the autoinhibited $\mathrm{N}$-terminal execution domain (NED) relieving its autoinhibition. Active MLKL is thought to form trimers or tetramers mediated by oligomerization of the regulatory brace. In conjunction with NED activation by IPs, NED associates with plasma membrane phospholipids promoting its rupture, although this mechanism is not understood. The ESCRT-III machinery orchestrates a general membrane repair mechanism antagonizing MLK-mediated plasma membrane rupture by shedding MLKL-containing plasma membrane 'bubbles' (not shown).

Excessive necroptosis may lead to diseases including neurodegeneration, cardiovascular disease, inflammatory bowel disease and multiple sclerosis [10]. Conversely, downregulation or loss of necroptosis may be responsible for tumor development in many different types of cancer, and for tumor resistance to chemotherapeutics [10]. The links between necroptosis and these diseases prompted interest in the discovery and development of small molecule modulators. Necroptosis inhibitors may be used when excessive necroptosis is causative, while necroptosis activators may be used to kill tumors. Being essential in necroptosis, RIPK3 and MLKL provide attractive drug targets in related pathophysiologies. Thus, elucidating their mechanisms of regulation is paramount to targeting them in diseases.

\section{Diverse necroptotic signals converge at RIPK3}

Three distinct, genetically defined means of activating RIPK3 in necroptosis have been discovered in mammals (reviewed in $[7,10]$ ). Canonical RIPK3 activation involves prototypical death receptor ligation of TNFR by TNF, which recruits RIPK1 to TNFR. To mediate cell death, including apoptosis and necroptosis, RIPK1 dissociates from the TNFR as part of a complex involving FADD and sometimes including TRADD (Figure 1). In cells exhibiting compromised caspase-8 activity or upon pharmacologic inhibition of caspase-8, the prodeath RIPK1-FADD and RIPK1-FADD-TRADD complexes recruit RIPK3 through amyloid-like interactions, which are mediated by RIP homotypic interaction motifs (RHIM) common to RIPK1 and RIPK3 (Figure 1). This leads to RIPK3 auto-phosphorylation and activation. Caspase-8 blocks RIPK3 activation by directly cleaving RIPK1 and RIPK3, 


\begin{tabular}{|c|c|c|}
\hline PDB & Structure & Ref. \\
\hline $4 \mathrm{btf}$ & Full-length mMLKL & [11] \\
\hline $4 m 66$ & mRIPK3 KD & [24] \\
\hline $4 m 67$ & hMLKL psKD & [24] \\
\hline $4 m 68$ & mMLKL psKD & [24] \\
\hline $4 m 69$ & mRIPK3 KD:mMLKL psKD complex & [24] \\
\hline $4 \mathrm{mwi}$ & hMLKL psKD & [23] \\
\hline $2 \mathrm{msv}$ & hMLKL NED & [22] \\
\hline $5 \mathrm{knj}$ & hMLKL psKD:compound 1 complex & [43] \\
\hline $5 \mathrm{ko1}$ & hMLKL psKD:compound 4 complex & [43] \\
\hline $6 \mathrm{bwk}$ & hMLKL psKD T357E S358E & [19] \\
\hline $6 \mathrm{~d} 74$ & hMLKL NED & [21] \\
\hline $6 \mathrm{ka} 4$ & Full-length AtMLKL3 tetramer & [25] \\
\hline
\end{tabular}

AtMLKL3: Arabidopsis thaliana MLKL3; hMLKL: Human MLKL; mMLKL: Mouse MLKL; NED: N-terminal executioner domain; mRIPK3: Mouse RIPK3; psKD: Pseudokinase domain.

thereby mitigating necroptosis signaling. RHIM-dependent interactions between RIPK3 and ZBP1, also known as DAI, and TRIF, also known as TICAM1, have been involved in noncanonical RIPK3 activation observed in certain cell types, including fibroblasts and endothelial cells upon dsRNA-induced necroptosis and cytomegalovirusinduced necroptosis, respectively $[7,10]$. The noncanonical RIPK3 activation pathways remain poorly investigated mechanistically.

\section{RIPK3 dynamically recruits MLKL promoting its activation through phosphorylation}

MLKL was discovered as an essential component of necroptosis downstream of RIPK3 (Figure 1) through chemical [5] and short hairpin RNA-mediated genetic screens [6] for loss-of-function phenotypes in TNF-induced necroptosis. Shortly thereafter, MLKL gene deletion in mouse was shown to completely abrogate necroptosis, which corroborated involvement of MLKL in this pathway [11,12]. Contrary to early reports, which hypothesized that MLKL signals downstream to other mechanisms of membrane rupture possibly involving ion channel-mediated osmotic shock [13,14], it has become accepted that RIPK3 and MLKL represents the penultimate and ultimate components of mammalian necroptosis, respectively [15]. Importantly, RIPK3 and MLKL are not essential genes for mouse physiology, as their deletion did not promote deleterious phenotypes [11,12,16]. Moreover, RIPK3 and MLKL are thought to have overlapping (necrotic) and nonoverlapping functions in pathophysiology, but how they are distinguished at the molecular level remains poorly understood [17].

Whereas RIPK3 and MLKL do not interact in healthy cells, biochemical studies have revealed that their interaction is absolutely essential in necroptosis, and their complex was named the necrosome (Figure 1). The RIPK3 kinase activity is required for their interaction and activation of MLKL in necroptosis of human and mouse cells, as kinase-dead RIPK3 mutants were unable to bind MLKL or mediate TNF-induced necroptosis [6,18]. Activated S227 autophosphorylated hRIPK3 and S231 and S232 autophosphorylated mRIPK3 have been shown to interact with hMLKL and mMLKL, respectively [5,18]. During necroptosis, RIPK3 phosphorylates T357 and S358 of hMLKL and S347 and T349 of mMLKL to promote activation of the pseudokinase (Figure 2A) [5,11]. Importantly, T357A S358A phospho site mutant hMLKL blocked the interaction with hRIPK3 and necroptosis [5,19]. The T357E S358D phosphomimetic hMLKL mutant also blocked interaction with hRIPK3, yet it induced spontaneous [5] or no necroptosis [19] in different cell types. The underlying reason for opposite outcomes in these studies may be high and low MLKL expression levels, respectively. These observations suggest that MLKL may kill cells even in the absence of RIPK3, provided it is triggered at high doses.

\section{Dormant MLKL: many ways to keep a killer at bay}

To understand how hMLKL is activated, we will compare and contrast human, mouse and emerging Arabidopsis thaliana (At)MLKL. The human RIPK3:MLKL necrosome currently remains incompletely characterized structurally and mechanistically (Table 1). Accordingly, we lack high-resolution structures for hRIPK3, with the exception of its amyloid core RHIM peptide [20]. Additionally, the structure of full-length hMLKL has not been solved, although its isolated N-terminal executioner domain (hNED) [21,22] and its pseudokinase domain (hpsKD) have 


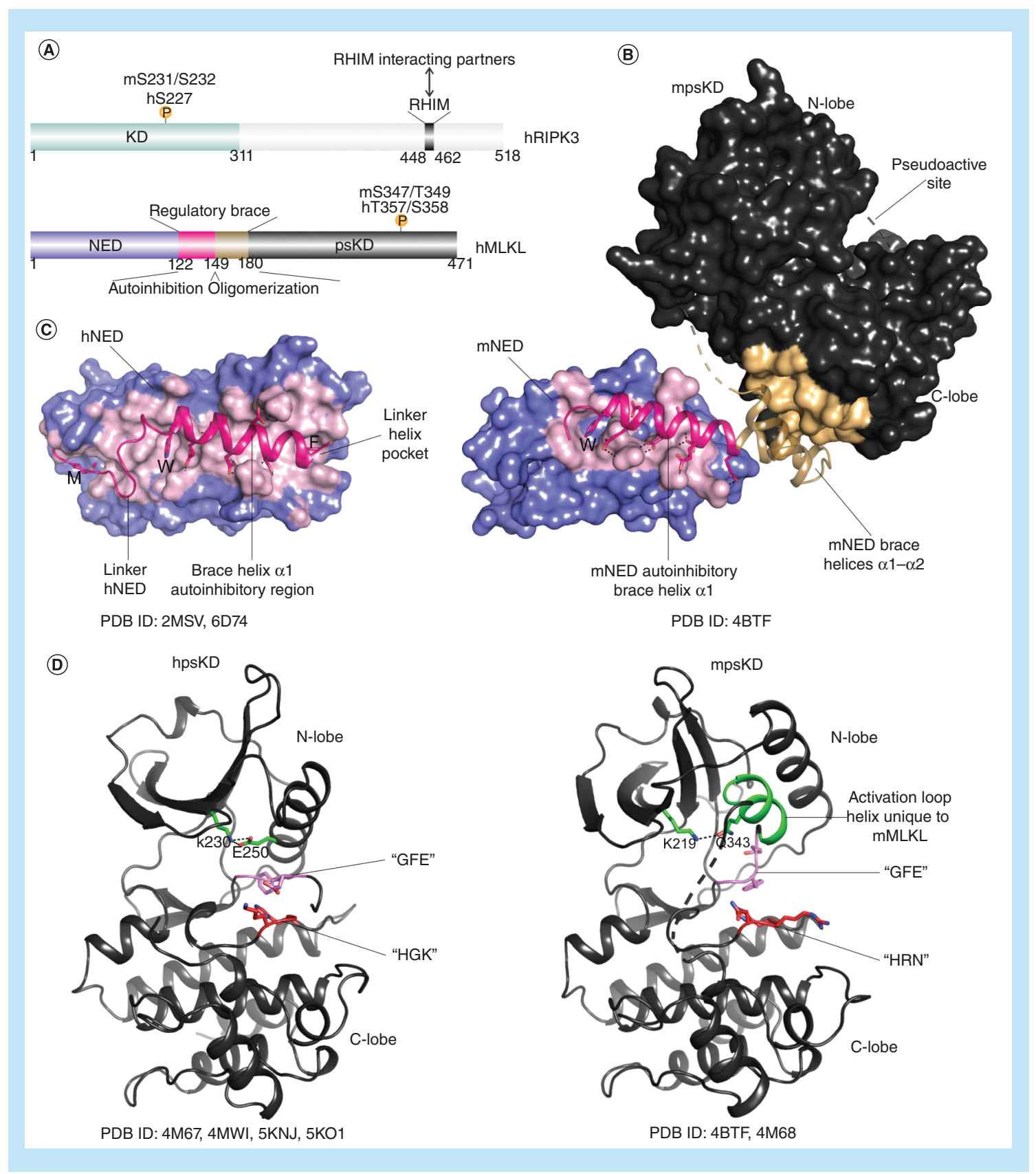

Figure 2. Domain organization and dormant structures of mammalian receptor interacting protein kinase 3 and mixed lineage kinase domain-like. (A) Human receptor interacting protein kinase 3 (hRIPK3) and human mixed lineage kinase domain-like (hMLKL) schematic indicating domain boundaries, their designations and functions. Regulatory brace connects the N- and C-terminal domains in MLKL contributing to autoinhibition and oligomerization. The human sequence contains additional residues, in the brace at the C-terminus of the autoinhibitory region, which may alter helicity compared with the mouse sequence. Phosphorylation sites critical for RIPK3 and MLKL activation are indicated for the human and mouse proteins. KD, kinase domain. (B) Mouse MLKL (mMLKL) crystal structure reveals the relative arrangement of the $\mathrm{N}$-terminal execution domain (NED), the regulatory brace, and the pseudokinase domain (psKD). The autoinhibitory brace is anchored to NED through an N-terminal tryptophan residue bound to a central hydrophobic pocket and electrostatic interactions from six acidic residues to basic residues of NED. The C-terminal region of the brace folds into a short helix-loop-helix motif interacting with a surface pocket on the psKD C-lobe. NED and psKD residues located $4 \AA$ from the brace are color coded on their surface representation revealing the extent of the interaction interfaces. The linker between NED and the brace helix $\alpha 1$ and the putative linker helix between NED helices $\alpha 3$ and $\alpha 4$ are intrinsically disordered in the mMLKL structure. (C) Autoinhibited hNED NMR structure reveals the linker and brace autoinhibitory region. The inhibitory linker is anchored to NED by a methionine and tryptophan. An additional NED linker helix creates a pocket to accommodate a phenylalanine at the C-terminus of the brace. Similar contacts seen in mMLKL stabilize autoinhibited hNED through the conserved tryptophan and 5 acidic-basic electrostatic interaction. NED residues located $4 \AA$ from the autoinhibitory region are color coded on the surface representation. (D) Crystal structures of human and mouse psKD reveal the pseudoactive site organization with the activation loop of the latter exhibiting a unique helix. Nucleotide binding (GFE) and active site motifs (HGK or HRN) are divergent from kinases (DFG and HRD, respectively) explaining the loss of kinase activity in MLKL. 


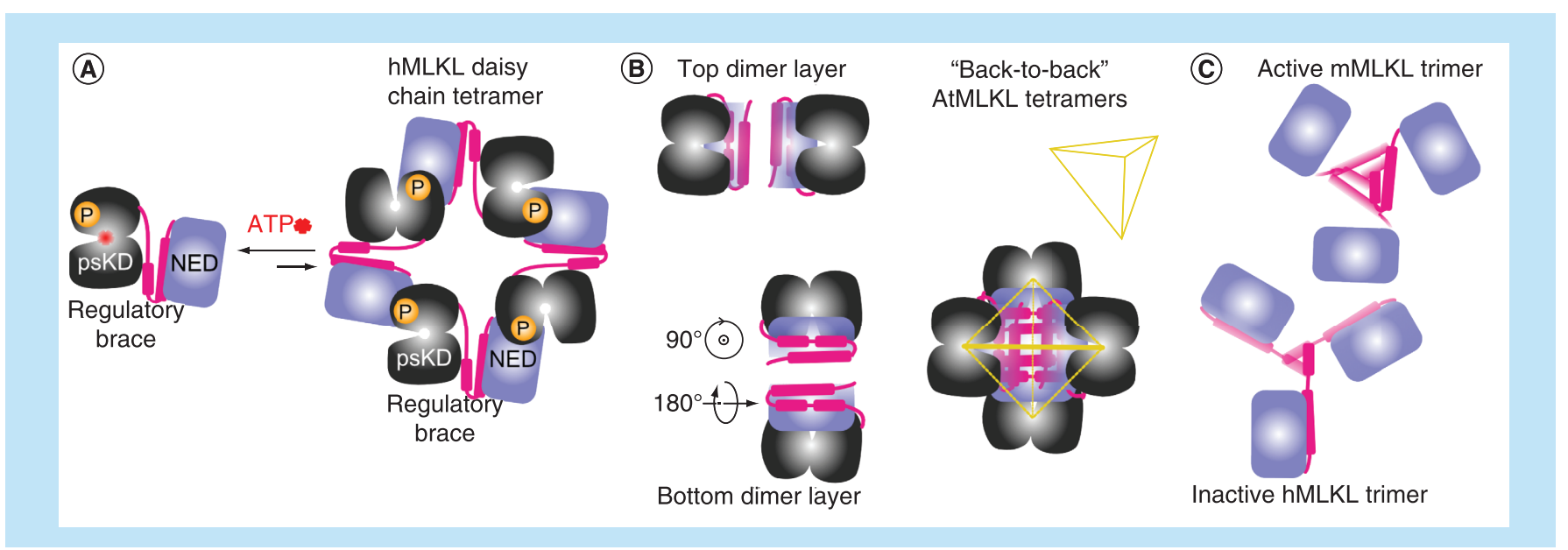

Figure 3. Quaternary mixed lineage kinase domain-like structures reveal different ways of N-terminal execution domain autoinhibition. (A) Low-resolution structural probing of human mixed lineage kinase domain-like (hMLKL) suggested a daisy chain tetramer organization with the N-terminal execution domain (NED) of one monomer binding to the pseudokinase domain (psKD) of another monomer. This arrangement does not reveal how NED autoinhibition may be relieved. The tetramer is in equilibrium with the monomer. ATP binding shifts the equilibrium in favor of the monomer. (B) The cryo-EM structures of Arabidopsis thaliana (At)MLKL2 and AtMLKL3 reveal a 'back-to-back' tetrahedral arrangement, wherein twofold dimers stabilized by an antiparallel NED-mediated interface are further stabilized by brace-mediated contacts at the center of the tetrameric assembly. Each of the four AtpsKDs in the tetramer are positioned at the vertices of a tetrahedron. Rotations of top dimer layer to achieve the bottom layer are indicated. The PDB ID for the unreleased cryo-EM structure of the AtMLKL3 tetramer is 6KA4. (C) Possible models of active mNED-brace and inactive hNED-brace constructs, involving trimerization of the brace region.

been determined (Figure 2) [23,24]. A caveat to interspecies comparisons is that the three pathways have been shown to be, as expected, quite divergent. For instance, mRIPK3 and hRIPK3 exclusively interact with the mMLKL and hMLKL, respectively [18]. Additionally, two divergent Arabidopsis thaliana mixed lineage kinase domain-like (AtMLKL) have recently been characterized [25], yet their RIPK3 upstream kinase counterparts have not been identified.

The crystal structure of monomeric full-length mMLKL reveals an $\mathrm{N}$-terminal helical domain connected by a regulatory region termed the brace to a C-terminal psKD (Figures $2 \mathrm{~A} \& \mathrm{~B}$ ) [11]. The mouse $\mathrm{N}$-terminal domain exhibits a bundle of 4 long helices, designated as the $4 \mathrm{HB}$ domain (4HBD). The m4HBD has two intrinsically disordered regions, one between helices $\alpha 3$ and $\alpha 4$ and another between $\alpha 4$ and $\alpha 1$ of the brace (Figure 2B). The corresponding domain in the human structure contains an additional short helix that links helices $\alpha 3$ and $\alpha 4$ of $\mathrm{m} 4 \mathrm{HBD}$ [21,22]. Sequence homology suggests that the mouse sequence may also adopt a helix in this linker [21]. The domain organization of mMLKL predicts clashes of the putative linker helix with the psKD domain, which may promote its unfolding. Interestingly, At $4 \mathrm{HBD}$, also named the HeLo domain, exhibits a linker helix similar to that seen in the h4HBD [25]. This domain was also termed the $\mathrm{N}$-terminal executioner domain (NED) because it is solely responsible for membrane rupture in necroptosis [21]. The hNED is autoinhibited by the brace helix $\alpha 1$ and its preceding linker, or the linker-brace autoinhibitory region (Figures $2 \mathrm{~A} \& \mathrm{C}$ ) $[21,22,26]$. The full-length hMLKL has been shown to exist in equilibrium between a monomer and a daisy chain tetramer, probed by low-resolution techniques including cross-linking and deuterium exchange mass spectrometry and small angle X-ray scattering (SAXS, Figure 3A, vide infra) [19].

Completely different structures have recently been determined for AtMLKL2 and AtMLKL3 by cryo-EM [25]. Both AtMLKL isoforms exhibit tetragonal caged structures with part of the brace and NED buried at the center and the psKD and remaining brace arranged at the vertices and periphery (Figure 3B). Each NED is partly sandwiched by the psKD and the brace. The AtMLKL monomers assemble into dimers with NED running antiparallel stabilized by a twofold helix $\alpha 1$-mediated interface. Two 'back-to-back' dimers rotated $90^{\circ}$ about the twofold axis assemble into tetramers (Figure 3B). The AtMLKL brace region is composed of five $\alpha$-helices that run peripheral to NED and interact extensively to stabilize the 'back-to-back' center of the tetramer. Contrary to the mouse and human arrangements, each AtNED is shielded by its brace, which interacts with NED helices $\alpha 2$ and $\alpha 4$, and by the psKD whose activation loop (vide infra) binds to NED helix $\alpha 3$. The psKD thus blocks access to a similar site 


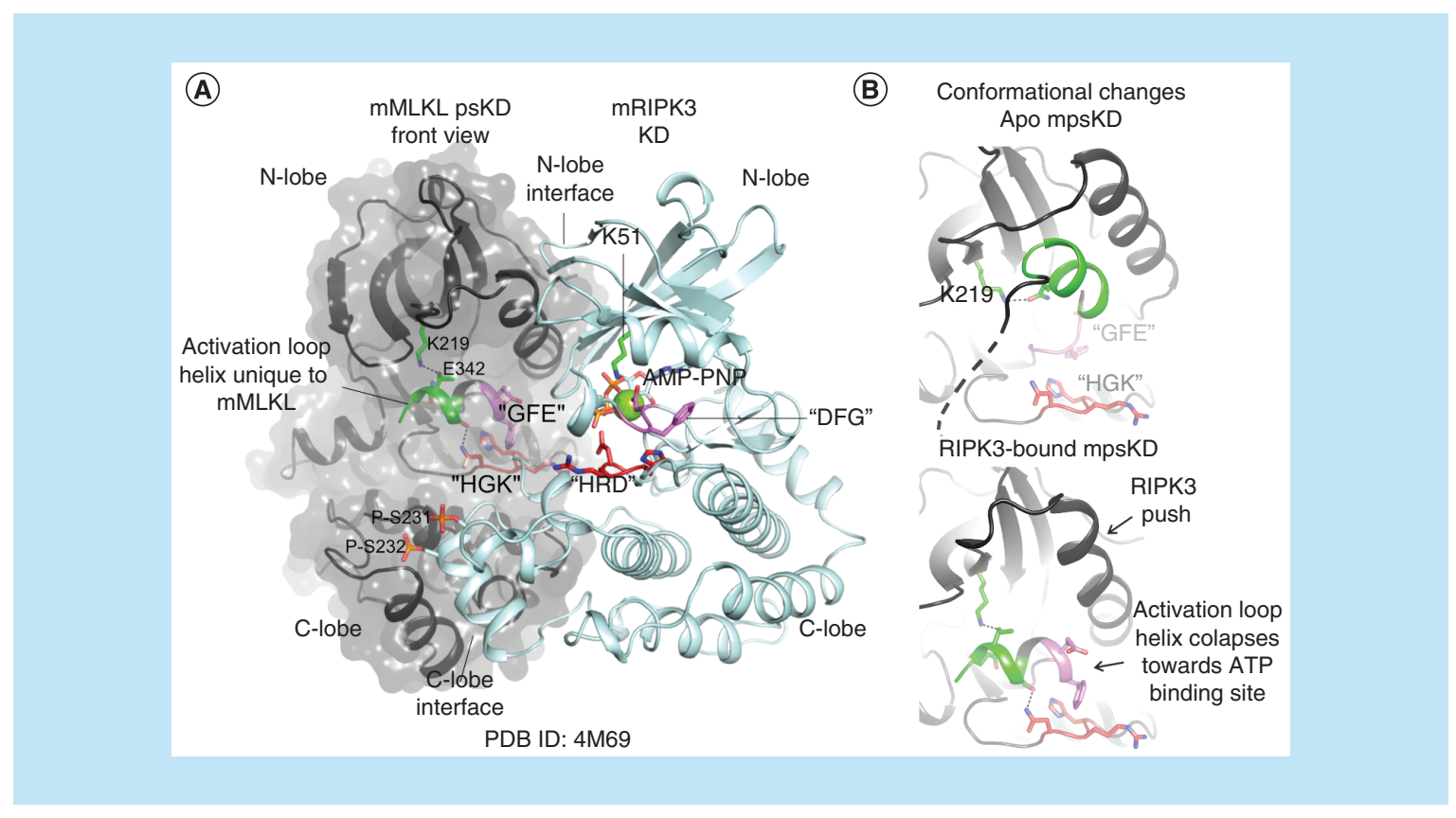

Figure 4. Structural basis of exquisite mouse receptor interacting protein kinase 3 selectivity for mouse mixed lineage kinase domain-like. (A) Crystal structure of mouse receptor interacting protein kinase 3 (mRIPK3):mouse mixed lineage kinase domain-like (mMLKL) kinase domain (KD):pseudokinase domain (psKD) complex revealed juxtaposition of mRIPK3 active site next to the pseudoactive site of mMLKL for phosphorylation of the latter's activation loop. This loop, which harbors the phosphorylation sites, is mostly intrinsically disordered and may easily protrude into the active site of mRIPK3 for phosphorylation. The KD:psKD complex is governed by extensive lobe-to-lobe interaction interfaces, stabilized by hydrophobic and electrostatic interactions. The C-lobe interface is mediated by RIPK3 autophosphorylated residues, explaining the exquisite selectivity of phospho-RIPK3 for MLKL. (B) Conformational changes induced by mRIPK3 KD binding to mMLKL psKD are extensive but confined to the N-lobe side of the pseudoactive site. Both $\alpha$-helices in the $\mathrm{N}$-lobe collapse toward the nucleotide binding site, which is partially occupied by the activation loop helix unique to mMLKL. The conformational changes in the C-lobe are minimal (not shown) and without additional information, such as provided by dynamics experiments, they do not explain how binding and phosphorylation may allosterically unleash the autoinhibitory brace from $\mathrm{N}$-terminal execution domain.

occluded by the brace helix $\alpha 1$ in the mouse and human MLKL. It is remarkable how divergent mechanisms of NED inactivation have evolved in different species, suggesting that their respective triggering mechanisms may be quite distinct.

\section{Kiss of death: how MLKL homes in on RIPK3 active site}

Although the complex between RIPK3 and MLKL is highly dynamic, governed by transient phosphorylationdependent interactions and heterogeneity of RIPK3 oligomerization [20,27], the mRIPK3:mMLKL KD:psKD heterodimer was determined by X-ray crystallography [24]. This complex did not form simply by mixing the two components; rather, it assembled when the components were co-expressed or when ATP was provided to their mixture in vitro. These strategies allowed RIPK3 autophosphorylation, which is essential for stable complex formation. If one considers the face of the psKD to be the pseudoactive site front view with the N-lobe and C-lobe at the top and bottom, respectively, RIPK3 appears to 'kiss' MLKL on the "left cheek" (or on the right side of the front view Figure $4 \mathrm{~A}$ ) in this complex. This orientation enables the presentation of MLKL activation loop to the RIPK3 active site for phosphorylation. The KD:psKD complex is stabilized by lobe-to-lobe interfaces with surface complementary established through extensive hydrophobic and hydrophilic interactions (Figure 4A). The C-lobe interface is additionally stabilized by electrostatic interactions mediated by RIPK3 phospho-Ser, PS231 and P-S232, potentially explaining why the two domains do not form stable complexes in the absence of phosphorylation (Figure 4A). This unique, exosite-driven kinase:substrate interaction ensures exquisite selectivity of active phospho-mRIPK3 for mMLKL.

The structure of the human RIPK3:MLKL complex has not been determined, but its modeling based on the mouse complex suggests that similar interaction may occur, governed by different electrostatic surface potentials. 
This may explain why the human and mouse RIPK3 and MLKL pairs do not cross-interact physically or functionally $[18,24]$. The regulation of AtMLKL isoforms is poorly understood, and the extent to which phosphorylation of their psKDs contributes to activation and cell death function has yet to be determined.

How MLKL phosphorylation by RIPK3 contributes to its activation remains a mystery. Overlay of apo mouse psKD with that of the RIPK3:MLKL complex revealed major conformational changes in this domain at its pseudoactive site (Figure $4 \mathrm{~B}$ ). The psKD activation loop harbors a unique $\alpha$-helix that moves into the position occupied by ATP in typical kinases, explaining why this domain is nucleotide-free in the mouse RIPK3:MLKL complex [24]. Additionally, the phosphorylation sites of the MLKL activation loop in this complex are intrinsically disordered, rendering them invisible in the crystal structure. Importantly, the region of interaction observed between the brace and psKD in the full-length mMLKL is largely indistinguishable in the RIPK3:MLKL complex. The lack in changes of conformation suggests that an allosteric effect mediated by the brace to NED is unlikely to be explained by the RIPK3-induced changes in the psKD of MLKL. Still, we cannot exclude the possibility of differences in the dynamics of this region contributing to an allosteric effect. Potentially more important than the RIPK3-mediated allosteric changes in the psKD, phosphorylation of MLKL by RIPK3 has been proposed to change the monomer-to-oligomer equilibrium in favor of the oligomer. The mechanism by which RIPK3 phosphorylation-dependent MLKL oligomerization manifests at the molecular level remains to be characterized and will ultimately reveal the direct effect, or lack thereof, on NED activation.

\section{MLKL oligomerization in resting cells \& during necroptosis}

It is now clear, based on the dormant monomeric mMLKL and the tetrameric caged AtMLKL structures, that monomers or oligomers should not necessarily be assumed to be the active species. Although structural information on any active MLKL species is lacking, we hypothesize that, so long as the brace helix $\alpha 1$ inhibits NED in hMLKL and mMLKL or the AtNED remains inactivated in a brace-NED-psKD sandwich seen in the AtMLKL monomers (Figures $2 \& 3$ ), these conformations are most likely inactive regardless of their monomeric or oligomeric arrangement $[21,22,26,28,29]$. With this in mind, purified mouse and human MLKL spontaneously adopt monomeric and oligomeric conformations that are affected by RIPK3-mediated phosphorylation or mutations of residues within their psKD [19,30]. The hMLKL apparently forms a daisy chain tetramer stabilized through interactions between NED of one monomer and psKD of another monomer (Figure 3A) [19]. In this tetramer, all NEDs face the same side of the structure, presumably being able to interact with the target membrane simultaneously [19]. Surprisingly, missense mutations in the autoinhibitory linker-brace region are able to spontaneously activate autoinhibited hNED (residues 1-140 or 1-156), which is monomeric, suggesting that its oligomerization is not

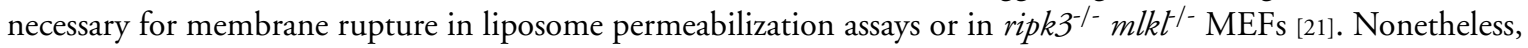
we believe that oligomerization may drive MLKL association with target membranes (vide infra) [26].

When discussing MLKL oligomerization one must make distinctions between dormant or active configurations as they relate to NED. It is apparent that NED is differentially inactivated in the mouse and human MLKL, with the latter being perhaps more tightly inhibited as suggested by liposome permeabilization assays with the respective purified proteins [21,31]. Interestingly, human and mouse MLKL constructs encoding NED-brace regions spontaneously trimerize, or possibly tetramerize for the human constructs, according to size exclusion chromatography and analytical ultracentrifugation (Figure 3C) [26,32]. However, the mouse construct is active in liposome permeabilization assays, whereas the human construct is inactive. Nonetheless, in $\mathrm{mlkt}^{/-}$MEFs or $m l k t^{/-}$mouse dermal fibroblasts (MDFs) the human and mouse NED-brace constructs are active, respectively. Modeling of the mNEDbrace trimer (1-169) by low-resolution SAXS analysis, suggested a radial trimer conformation in solution [32]. Interestingly, in this model the brace helix $\alpha 1$ region is displaced from NED and participates in trimerization, indirectly suggesting that NED is freed and therefore active, although it is unclear how the displacement from NED of the putative autoinhibitory region occurs (Figure 3C) [32]. On the other hand, preliminary nuclear magnetic resonance (NMR) spectroscopy analysis of a hNED-brace construct spanning residues 2-178 indicated a largely overlapping ${ }^{1} \mathrm{H}_{-}{ }^{15} \mathrm{~N} 2 \mathrm{D}$ NMR spectrum with that of the autoinhibited NED structure (2-154), suggesting that the brace helix $\alpha 1$ probably assumes the same position in both structures (Figure 3C) [22]. This is consistent with the hNED-brace and autoinhibited hNED constructs being resistant to spontaneous membrane permeabilization in vitro [21,31]. Future, high-resolution structures of human and mouse NED-brace constructs will resolve these discrepancies.

MLKL tetramers and octamers were detected in cells undergoing TNF-mediated necroptosis through Cysoxidation under nonreducing conditions and by crosslinking analyses [33]. Apparently, the octamers form before 
association with membranes. Once associated with target membranes through NED, active MLKL may adopt yet other membrane-bound oligomeric conformations awaiting characterization. It remains to be seen if the brace couples NED to psKD structurally in active human and mouse MLKL. It is worth noting that upon dissociation from NED by inositol phosphate metabolites or through lipid binding, the brace helix $\alpha 1$ becomes intrinsically disordered in isolated, autoinhibited NED constructs [21,26,28,29].

\section{Metabolic regulation of human MLKL}

ATP is a negative regulator of MLKL

The MLKL psKD binds adenosine nucleotides and analogs in a divalent cation-independent manner $[11,19]$. ATP binds isolated psKD with a $K_{\mathrm{D}}$ of $\sim 30 \mu \mathrm{M}$; consequently, MLKL psKD should readily bind ATP in cells given typically high 1-10 mM ATP levels reported for most cells. Although the mode of binding to MLKL has not been revealed at high resolution, ATP has been shown to modulate oligomerization and membrane permeabilization by MLKL [19]. Purified WT FL hMLKL, which exhibits both monomeric ( $30 \%$ of total) and tetrameric ( $70 \%$ of total) species, and the $\mathrm{K} 331 \mathrm{~N}$ mutant, which is exclusively tetrameric, have essentially the same activity in liposome permeabilization [19]. One interpretation of these data is that tetramerization does not contribute significantly to membrane permeabilization, since the K331N mutant should have been more active than the WT, assuming that these tetramers indeed represent active MLKL. Nonetheless, ATP shifted the equilibrium of WT FL hMLKL toward the monomeric state ( $70 \%$ of total) and decreased its ability to permeabilize liposomes (Figure 3A) [19]. In contrast, ATP did not affect spontaneous tetramerization and marginally decreased K331N MLKL activity [19]. This suggests that ATP negatively regulates MLKL activity by controlling its oligomerization.

\section{Inositol phosphate metabolites activate human MLKL}

Autoinhibited hNED and full-length hMLKL are not spontaneously active in functional liposome permeabilization assays, but they are activated by a select subset of inositol phosphate metabolites, including $\mathrm{I}(1,3,4,6) \mathrm{P}_{4}\left(\mathrm{IP}_{4}\right)$, $\mathrm{I}(1,3,4,5,6) \mathrm{P}_{5}\left(\mathrm{IP}_{5}\right)$ and $\mathrm{IP}_{6}[21]$. This is consistent with the recent discovery of the IPKs IPMK and ITPK1 as essential regulators of MLKL-mediated necroptosis in a forward genetic screen performed with the human haploid cell line HAP1 [28]. Subsequent genetic deletion of IPK genes ipmk, itpk1 and $i p p k$ of the IP code metabolic pathway - which phosphorylate inositol $(1,4,5)$ triphosphate $\left(\mathrm{IP}_{3}\right)$ to the higher order activators of MLKL IP 4 , $\mathrm{IP}_{5}$ and $\mathrm{IP}_{6}$, blocked MLKL-mediated necroptosis in HT-29 human colon adenocarcinoma cells [21,28].

$\mathrm{IP}_{4}, \mathrm{IP}_{5}$ and $\mathrm{IP}_{6}$ act in concert to directly interact and activate hNED (Figure 5A) [21,28]. They do so by binding up to three distinct structural sites along autoinhibited hNED. IP engagement is thought to reshuffle selective NED amino acid side chains within these sites, releasing the autoinhibitory linker-brace region, and liberating NED for membrane permeabilization. The IP binding sites of hNED were mapped by NMR, which showed them to be rich in amino acids that have previously been implicated in IP coordination in other proteins. Based on the hyperbolic nature of their binding curves, these sites appear to independently bind $\mathrm{IP}_{4}, \mathrm{IP}_{5}$ or $\mathrm{IP}_{6}$ with $\mathrm{K}_{\mathrm{D}}$ values of $100-600 \mu \mathrm{M}$, a range that overlaps the high end of their cellular levels $(5-100 \mu \mathrm{M})$. Exactly how these sites bind IPs remains to be elucidated at high resolution. It is currently unknown if IPs activate mMLKL or AtMLKL. Interestingly, CRISPR whole-genome screening of L929 mouse fibroblasts, aimed at identifying new mouse necroptosis regulators, has not revealed mouse IPKs among the significant hits [34]. In order to validate the IP-mediated autoinhibitory region release, missense mutations in the NED autoinhibitory linker-brace region were shown to spontaneously activate hNED in vitro and in cells, bypassing the need for activating IP metabolites in liposome permeabilization (vide infra) [21,22,26]. It is highly possible that MLKL activation prior to plasma membrane rupture must ultimately elicit the release of the autoinhibitory region and NED activation. Thus, the IPs provide the missing link in this process.

\section{Lipid binding \& plasma membrane rupture by hMLKL}

During necroptosis, active MLKL is passively recruited to target membranes to permeabilize them. Early on during this process cations may leak through the plasma membrane in an MLKL-dependent manner, as indicated from electrophysiology studies suggesting that MLKL forms cation channels [35]. As the process progresses, MLKL elicits rupture of the plasma membranes, although the mechanism remains elusive. Membrane targeting is facilitated by NED binding to specific phospholipids, including several phosphatidylinositol phosphates (PIPs), phosphatidylserine (PS) and cardiolipin $[26,30,36]$. Biochemical fractionation of cellular membranes revealed that all membranes contain MLKL in cells undergoing necroptosis [30]. However, strong evidence was provided for plasma membrane 


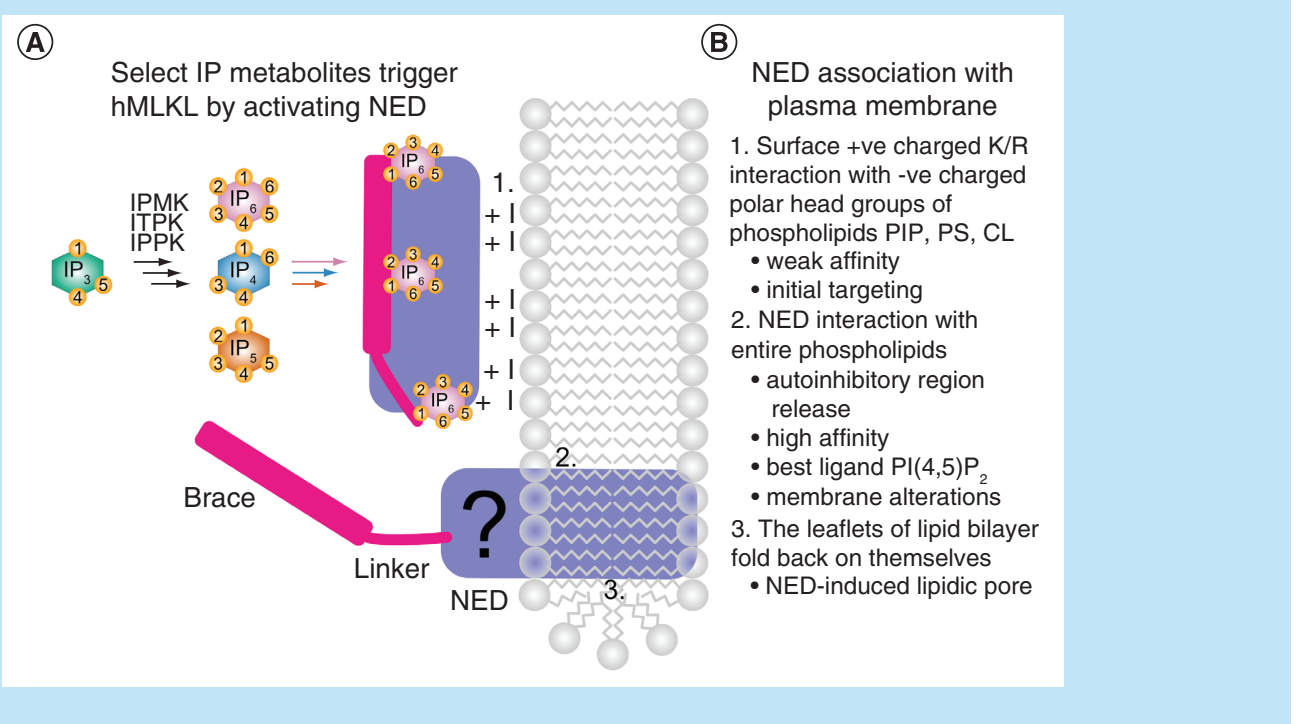

Figure 5. Inositol phosphate code of mixed lineage kinase domain-like activation and membrane association. (A) The autoinhibited N-terminal execution domain (NED) of human mixed lineage kinase domain-like (hMLKL) is activated by inositol phosphate metabolites IP4, IP5 and IP6 produced by the IPKs inositol phosphate multikinase (IPMK), inositol tetrakisphosphate kinase 1 (ITPK1) and inositol pentakisphosphate 2-kinase (IPPK). Activating IPs act in concert by binding three distinct sites along autoinhibited NED to destabilize the autoinhibitory linker-brace region. In the presence of membranes, the linker-brace autoinhibitory region is displaced allowing NED membrane targeting. It is unknown if this mechanism is conserved for mMLKL and Arabidopsis thaliana (At)MLKL. (B) MLKL NED exhibits two levels of interaction with membranes. 1) To home in onto the plasma membrane, positively charged residues of NED located on its opposite face to the autoinhibitory region weakly interact with negatively charged polar head groups of a select subset of phospholipids. 2) With help from activating IP metabolites, the autoinhibitory region is fully released facilitating high affinity phospholipid binding. This second membrane associating event likely leads to membrane alterations. The structures of NED associated with membranes are unknown. Subsequently, 3) membrane rupture ensues through putative lipidic pores, whereby the two leaflets of the lipid bilayer fold back on themselves.

recruitment and rupture by MLKL based on total internal reflection fluorescence microscopy (TIRFM), which can detect plasma membrane-associated processes, and 3D scanning electron microscopy (SEM), which can detect plasma membrane lesions [26]. To capture MLKL-mediated plasma membrane rupture, a fast-kinetics necroptosis assay was developed based on drug-induced dimerization of hNED [26,29]. In this assay, rapid accumulation of hNED to the plasma membrane, observed by TIRFM, preceded plasma membrane alterations, including micro and macro ruptures, observed by 3D SEM. How rupture of other cellular membranes contributes to necroptosis remains to be demonstrated.

The ESCRT-III machinery acts as a general membrane repair pathway antagonizing MLKL-mediated plasma membrane alterations and shedding so called MLKL-containing plasma membrane 'bubbles' [37]. This delays necroptosis, allowing parallel pathway to exert additional immune signaling functions including cytokine production and antigen cross-priming of $\mathrm{CD}^{+} \mathrm{T}$ cells [37]. This machinery was also implicated in downregulation of pyroptosis, another form of immunogenic cell death, by shedding of plasma membrane gasdermin $\mathrm{D}$ pores through a similar mechanism [38].

We propose a revised interaction of hMLKL with the plasma membrane (Figure 5B). Positively charged surface residues of hNED, including K5, K16, R17, K50, R51 on the face opposite the autoinhibitory region, and possibly others, bind negatively charged polar head groups of membrane phospholipids with low affinity, aided by bracemediated oligomerization to promote avidity for the plasma membrane (Figure 1) [26]. Membrane-associated hNED cooperates with activating IP metabolites $\mathrm{IP}_{4}, \mathrm{IP}_{5}$ and $\mathrm{IP}_{6}$ to release the autoinhibitory linker-brace region [21,28]. This facilitates potent binding of activated hNED to phospholipids, especially its best ligand, $\mathrm{PI}(4,5) \mathrm{P}_{2}[26,30,36]$. Remarkably, phospholipids in detergent micelles also have the ability to release the autoinhibitory linker-brace from hNED, similarly to activating IPs [26], although they are unable to do so when they are embedded in membranes [21]. It has been suggested that hMLKL induces lipidic pores as a result of bilayers folding back on themselves [10,39]. 


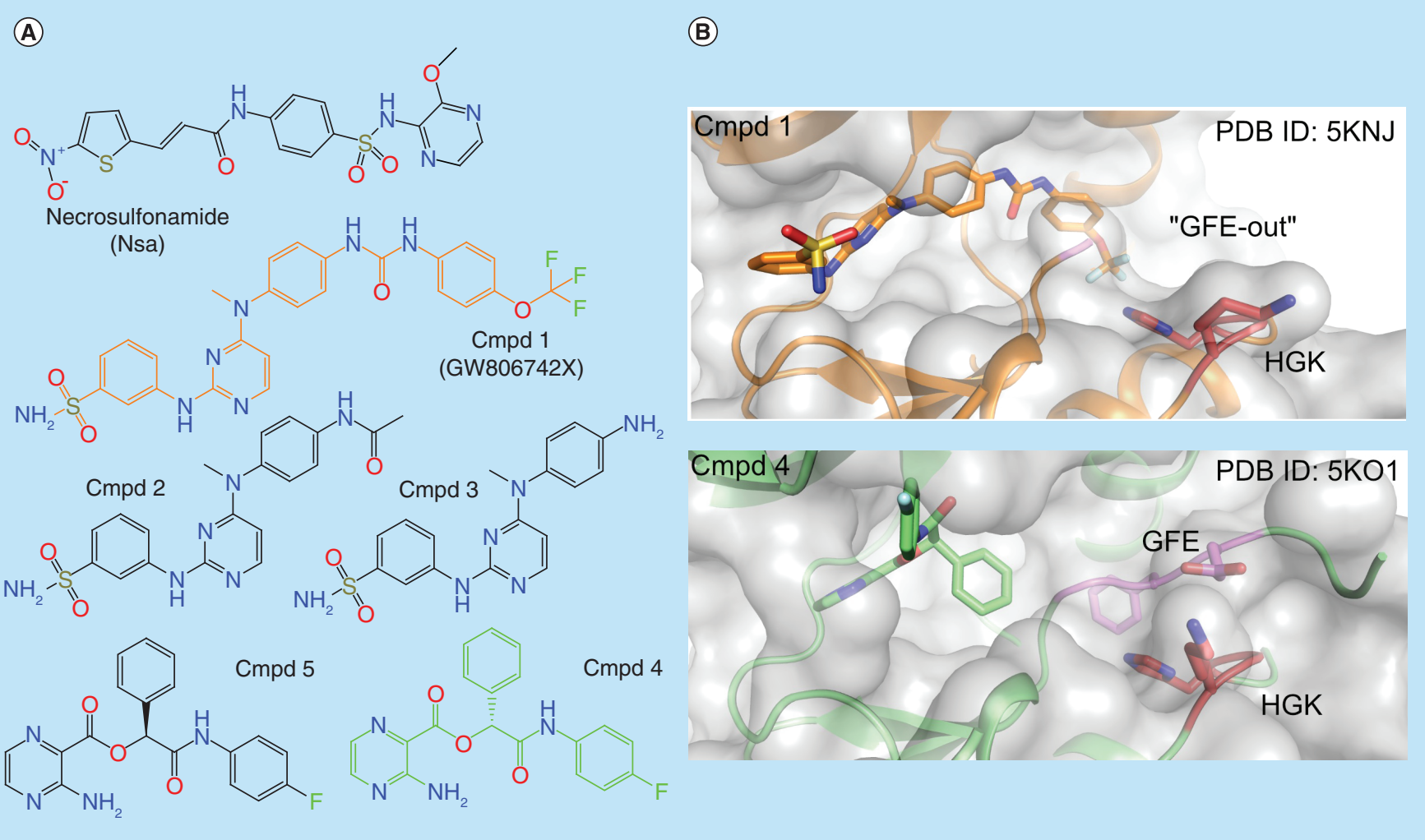

Figure 6. Mixed lineage kinase domain-like (nonselective) chemical probes. (A) Chemical structures of mixed lineage kinase domain-like (MLKL) targeting compounds. Necrosulfonamide targets C86 of human MLKL (hMLKL), which is positioned in the N-terminal execution domain linker helix. Compound 1 is a nonselective kinase inhibitor that binds MLKL and cross-reacts with receptor interacting protein kinase 1, receptor interacting protein kinase 3, and other kinases. Compound 4 is an exquisitely selective MLKL binder, yet it does not block MLKL-mediated necroptosis. These data indicate that ATP site binders may not be fruitful in inhibiting MLKL activity in necroptosis. (B) Crystal structures of compounds 1 and 4 bound to hMLKL pseudokinase domain. Compound 1 induces a typical 'GFE-out' pseudoactive site conformation. Compound-induced conformational changes are restricted to the pseudoactive site. Key pseudoactive site residues discussed in previous figures are labeled.

Exactly how these pores form is unclear but possible mechanisms have been discussed in a recent review [10]. The high-resolution details of hMLKL association with and rupture of membranes await characterization, as do the details of these processes for mouse and plant MLKL.

\section{Toward modulation of MLKL-mediated necroptosis with small molecules}

Kinase inhibitors designed for RIPK1 and RIPK3 have been established as selective chemical probes (reviewed in $[7,40])$. Nonselective MLKL chemical probes with cell activity have also been discovered (Figure $6 \&$ Table 2). Necrosulfonamide (Nsa; IUPAC names of compounds may be found in Table 2), the compound originally found to block MLKL-mediated necroptosis, is an irreversible cysteine-targeting compound that tethers by Michael addition to C86 of hMLKL (Figure 6A \& Table 2). C86 is located in the linker helix of hNED. The structural basis of Nsamediated inhibition of hMLKL is unknown, but testing of truncated forms of hMLKL indicated that Nsa requires NED and an intact brace region for inhibition, as shorter NED constructs were resistant to Nsa inhibition [26]. Unfortunately, Nsa cannot inhibit mMLKL because the mouse protein lacks C86. Additionally, Nsa is nonselective also targeting gasdermin D [41], and probably other proteins.

Compounds that reversibly target the nucleotide binding site of hMLKL psKD have been discovered [42-44] (Figure 6A \& Table 2). Compound 1 ( $\mathrm{cmpd} \mathrm{1)}$ was originally reported to inhibit MLKL and necroptosis [42], but later was characterized as nonselective also inhibiting RIPK1 and RIPK3 activities in addition to many other human kinases [43] (Table 2). The crystal structure of hpsKD in complex with cmpd 1 revealed binding at the putative nucleotide binding site in the 'GFE-out' conformation seen with type II kinase inhibitors (Figure 6B) [43]. 
Table 2. Binding, selectivity and activity of mixed lineage kinase domain-like targeted small molecules.

\begin{tabular}{|c|c|c|c|c|c|}
\hline Compound & MLKL $K_{D}(n M)$ & Fold selectivity over RIPK1 & Fold selectivity over RIPK3 & Necroptosis $I_{50}(\mu \mathrm{M})$ & Ref. \\
\hline Nsa & NA & NA & NA & 0.2 & {$[5,43]$} \\
\hline Cmpd 1 & 116 & 3.2 & 6.5 & 1.85 & {$[42,43]$} \\
\hline Cmpd 3 & 880 & $>11.4$ & NA & $>40$ & [43] \\
\hline Cmpd 4 & 230 & $>130.4$ & $>130.4$ & $>40$ & [43] \\
\hline
\end{tabular}

Nsa, (E)-N-(4-(N-(3-methoxypyrazin-2-yl)sulfamoyl)phenyl)-3-(5- nitrothiophene-2-yl)acrylamide.

Cmpd 1, 3-\{4-[methyl(\{2-[(3-sulfamoylphenyl)amino]pyrimidin-4-yl\})amino]phenyl\}-1-[4-(trifluoromethoxy)phenyl]urea.

Cmpd 2, N-\{4-[(2-\{[3-(aminosulfonyl)phenyl]amino $\}$ pyrimidin-4-yl)(methyl)-amino]phenyl $\}$ acetamide.

Cmpd 3, 3-(\{4-[(4-aminophenyl)(methyl)amino]pyrimidin-2-yl\}amino)benzene-sulfonamide.

Cmpd 4, 3-Amino-pyrazine-2-carboxylic acid (R)-(4-fluorophenylcarbamoyl)-phenyl-methyl ester.

Cmpd 5, 3-Amino-pyrazine-2-carboxylic acid (S)-(4-fluorophenylcarbamoyl)-phenyl-methyl ester.

MLKL: Mixed lineage kinase domain-like; NA: Not applicable; RIPK3: Receptor interacting protein kinase 3.

Two analogs of cmpd 1 lacking the urea phenyl trifluoromethyl ester moiety, cmpd 2 and cmpd 3, are expected to be type I binders being too short to induce the GFE-out conformation (Figure 6A \& Table 2) [43]. Cmpd 2 and cmpd 3 exhibit better selectivity for MLKL over RIPK1 and RIPK3, yet they exhibit no cell-based activity in necroptosis [43]. Another small molecule, cmpd 4, was discovered as an exquisitely selective type I hpsKD binder $\left(\mathrm{K}_{\mathrm{D}}\right.$ of $230 \mathrm{nM},>130$-fold selective over RIPK1 and RIPK3; Figure 6 \& Table 2), whereas its enantiomer, cmpd 5, is not potent or selective for MLKL (Figure 6A \& Table 2) [43]. Unfortunately, cmpd 4 did not exhibit any effect on the necroptosis pathway [43]. These observations reinforce the interpretation for the effect of ATP in MLKL oligomerization and membrane permeabilization by suggesting that endogenous or synthetic ATP-pocket MLKL binders are unable to allosterically displace the autoinhibitory region to activate NED despite possibly regulating MLKL oligomerization.

Discovery of the IPKs IPMK and ITPK1 as indispensable components of human necroptosis reveals an innovative alternative for targeting MLKL by depleting essential IP metabolites required for its activation $[21,28]$. This should stimulate drug discovery efforts aimed at targeting these IPKs in necroptosis [45]. Although IPMK [46] and most likely ITPK1 [47] are essential genes, their pharmacologic inhibition may be well tolerated in vivo. This is the case with targeting the anti-apoptotic BCL-2 protein MCL-1, which is also an essential gene [48], yet its selective inhibitors, such as $\mathrm{S} 63845$ [49], are well tolerated in vivo.

\section{Future perspective}

Despite great progress, understanding hMLKL regulation mechanistically remains in its infancy. Elucidating high-resolution structures of additional hMLKL conformations, including full-length dormant, RIPK3-associated, IP-associated, active and membrane-associated states, is necessary to fully characterize hMLKL activation mechanistically. This information will more effectively guide drug discovery efforts aimed at targeting necroptosis in pathophysiology. To our knowledge, hMLKL is the first example of a cell death pathway core component that is a sensor and effector of signaling (IP) metabolites [21,28,50]. We do not fully appreciate why MLKL evolved these metabolic features, but this area of research is potentially one of the most exciting emerging aspects of necroptosis biology. Future work will reveal if the IP regulation of hMLKL activation is a general mechanism in mouse, plants and related species. Understanding how the IP levels fluctuate and are modulated in pathophysiology to activate MLKL in necroptosis is an unexplored area that may reveal new biology of the IP metabolic pathway regulation in necroptosis.

\section{Open access}

This work is licensed under the Creative Commons Attribution-NonCommercial 4.0 Unported License. To view a copy of this license, visit http://creativecommons.org/licenses/by-nc-nd/4.0/ 
Financial \& competing interests disclosure

This work was supported by American Lebanese Syrian Associated Charities and St. Jude Children's Research Hospital. The authors have no other relevant affiliations or financial involvement with any organization or entity with a financial interest in or financial conflict with the subject matter or materials discussed in the manuscript apart from those disclosed.

No writing assistance was utilized in the production of this manuscript.

\section{Executive summary}

- Necroptosis is a mammalian immunogenic cell death program implicated in multiple pathophysiologies.

- Mixed lineage kinase domain-like (MLKL), the executioner of plasma membrane rupture in necroptosis, is an autoinactivated fusion of a pore-forming domain and a pseudokinase domain.

- The receptor interacting protein kinase 3 activates MLKL through phosphorylation promoting its oligomerization.

- The inositol phosphate kinases IPMK, ITPK1 and IPPK synthesize essential activators of human MLKL - IP, $\mathrm{IP}_{5}$ and $\mathrm{IP}_{6}$.

- Structural analyses have provided critical insights into the autoinactivation of the human, mouse and plant MLKL dormant structures.

- The structure of the mouse receptor interacting protein kinase 3:MLKL kinase domain:pseudokinase domain complex revealed its exquisite selectivity but no insights into how phosphorylation activates MLKL.

- Structural insights into MLKL activation and membrane permeabilization are needed.

- Opportunities for modulating MLKL-mediated necroptosis in related diseases include targeting its kinase regulators and MLKL itself.

\section{References}

Papers of special note have been highlighted as: $\bullet$ of interest; $\bullet \bullet$ of considerable interest

1. Tang D, Kang R, Berghe TV, Vandenabeele P, Kroemer G. The molecular machinery of regulated cell death. Cell Res. 29(5), 347-364 (2019).

2. Cho YS, Challa S, Moquin D et al. Phosphorylation-driven assembly of the RIP1-RIP3 complex regulates programmed necrosis and virus-induced inflammation. Cell 137(6), 1112-1123 (2009).

3. He S, Wang L, Miao L et al. Receptor interacting protein kinase-3 determines cellular necrotic response to TNF-alpha. Cell 137(6), 1100-1111 (2009).

4. Zhang DW, Shao J, Lin J et al. RIP3, an energy metabolism regulator that switches TNF-induced cell death from apoptosis to necrosis. Science 325(5938), 332-336 (2009).

5. Sun L, Wang H, Wang $\mathrm{Z}$ et al. Mixed lineage kinase domain-like protein mediates necrosis signaling downstream of RIP3 kinase. Cell 148(1-2), 213-227 (2012).

-• Landmark study discovering mixed lineage kinase domain-like (MLKL) in a chemical screen as a core component of necroptosis downstream of receptor interacting protein kinase 3 (RIPK3).

6. Zhao J, Jitkaew S, Cai $Z$ et al. Mixed lineage kinase domain-like is a key receptor interacting protein 3 downstream component of TNF-induced necrosis. Proc. Natl Acad. Sci. USA 109(14), 5322-5327 (2012).

- Study discovering MLKL in a genetic screen as a core component of necroptosis downstream of RIPK3.

7. Galluzzi L, Kepp O, Chan FK, Kroemer G. Necroptosis: mechanisms and relevance to disease. Annu. Rev. Pathol. 12, 103-130 (2017).

8. Wallach D, Kang TB, Dillon CP, Green DR. Programmed necrosis in inflammation: toward identification of the effector molecules. Science 352(6281), aaf2154 (2016).

9. Weinlich R, Oberst A, Beere HM, Green DR. Necroptosis in development, inflammation and disease. Nat. Rev. Mol. Cell. Biol. 18(2), 127-136 (2017).

10. Grootjans S, Vanden Berghe T, Vandenabeele P. Initiation and execution mechanisms of necroptosis: an overview. Cell. Death Differ 24(7), 1184-1195 (2017)

-• Comprehensive review of the mechanisms of necroptosis activation and execution in health and disease, providing possible mechanism of plasma membrane rupture by MLKL.

11. Murphy JM, Czabotar PE, Hildebrand JM et al. The pseudokinase MLKL mediates necroptosis via a molecular switch mechanism. Immunity 39(3), 443-453 (2013).

-. Landmark study revealing the structure of mouse MLKL and the phenotypes of MLKL gene deletion in mouse and in cells.

12. Wu J, Huang Z, Ren J et al. Mlkl knockout mice demonstrate the indispensable role of Mlkl in necroptosis. Cell Res. 23(8), 994-1006 (2013). 
13. Cai Z, Jitkaew S, Zhao J et al. Plasma membrane translocation of trimerized MLKL protein is required for TNF-induced necroptosis. Nat. Cell Biol. 16(1), 55-65 (2014).

14. Chen X, Li W, Ren J et al. Translocation of mixed lineage kinase domain-like protein to plasma membrane leads to necrotic cell death. Cell Res. 24(1), 105-121 (2014).

15. Petrie EJ, Czabotar PE, Murphy JM. The structural basis of necroptotic cell death signaling. Trends Biochem. Sci. 44(1), 53-63 (2019).

- Comprehensive review of the structural basis of necroptosis.

16. Newton K, Sun X, Dixit VM. RIP3 is dispensable for normal NF-kappa Bs, signaling by the B-cell and T-cell receptors, tumor necrosis factor receptor 1, and Toll-like receptors 2 and 4. Mol. Cell. Biol. 24(4), 1464-1469 (2004).

17. Newton K, Dugger DL, Maltzman A et al. RIPK3 deficiency or catalytically inactive RIPK1 provides greater benefit than MLKL deficiency in mouse models of inflammation and tissue injury. Cell. Death Differ. 23(9), 1565-1576 (2016).

18. Chen W, Zhou Z, Li L et al. Diverse sequence determinants control human and mouse receptor interacting protein 3 (RIP3) and mixed lineage kinase domain-like (MLKL) interaction in necroptotic signaling. J. Biol. Chem. 288(23), 16247-16261 (2013).

19. Petrie EJ, Sandow JJ, Jacobsen AV et al. Conformational switching of the pseudokinase domain promotes human MLKL tetramerization and cell death by necroptosis. Nat. Commun. 9(1), 2422 (2018).

- Study demonstrating the possible mechanism of tetramerization of human MLKL (hMLKL).

20. Mompean M, Li W, Li J et al. The structure of the necrosome RIPK1-RIPK3 core, a human hetero-amyloid signaling complex. Cell 173(5), 1244-1253; e1210 (2018).

21. McNamara DE, Dovey CM, Hale AT et al. Direct activation of human MLKL by a select repertoire of inositol phosphate metabolites. Cell Chem. Biol. 26(6), 863-877; e867 (2019).

- Study demonstrating the activation of hMLKL by inositol phosphate metabolites and a more extensive autoinhibitory region of human N-terminal execution domain (hNED).

22. Su L, Quade B, Wang H, Sun L, Wang X, Rizo J. A plug release mechanism for membrane permeation by MLKL. Structure 22(10), 1489-1500 (2014).

-• Study determining the first NMR structure of autoinhibited hNED to propose the autoinhibitory mechanism of hNED by the brace.

23. Murphy JM, Lucet IS, Hildebrand JM et al. Insights into the evolution of divergent nucleotide-binding mechanisms among pseudokinases revealed by crystal structures of human and mouse MLKL. Biochem. J. 457(3), 369-377 (2014).

24. Xie T, Peng W, Yan C, Wu J, Gong X, Shi Y. Structural insights into RIP3-mediated necroptotic signaling. Cell. Rep. 5(1), 70-78 (2013).

-• Structure-function study revealing the mechanism of exquisite selectivity of mRIPK3 for mMLKL.

25. Mahdi L, Huang M, Zhang X et al. Plant mixed lineage kinase domain-like proteins limit biotrophic pathogen growth. bioRxiv https://doi.org/10.1101/681015 (2019).

- Study detailing the dormant structure and function of AtMLKLs in plants.

26. Quarato G, Guy CS, Grace CR et al. Sequential engagement of distinct MLKL phosphatidylinositol-binding sites executes necroptosis. Mol. Cell. 61(4), 589-601 (2016).

-• Study revealing sequential steps in hMLKL activation and plasma membrane association and rupture during necroptosis.

27. Raju S, Whalen DM, Mengistu M et al. Kinase domain dimerization drives RIPK3-dependent necroptosis. Sci. Signal. 11(544), pii: eaar2188. (2018).

28. Dovey CM, Diep J, Clarke BP et al. MLKL requires the inositol phosphate code to execute necroptosis. Mol. Cell. 70(5), 936-948; e937 (2018).

-• Landmark study of the discovery of IPMK and ITPK1 as essential genes in human necroptosis through synthesis of IP metabolites as MLK activators.

29. McNamara DE, Quarato G, Guy CS, Green DR, Moldoveanu T. Characterization of MLKL-mediated plasma membrane rupture in necroptosis. J. Vis. Exp. (In Press), e58088 (2018).

30. Wang H, Sun L, Su L et al. Mixed lineage kinase domain-like protein MLKL causes necrotic membrane disruption upon phosphorylation by RIP3. Mol. Cell. 54(1), 133-146 (2014).

- Study revealing the phospholipid-binding and membrane permeabilization by MLKL.

31. Tanzer MC, Matti I, Hildebrand JM et al. Evolutionary divergence of the necroptosis effector MLKL. Cell Death Differ. 23(7), 1185-1197 (2016).

32. Davies KA, Tanzer MC, Griffin MDW et al. The brace helices of MLKL mediate interdomain communication and oligomerisation to regulate cell death by necroptosis. Cell Death Differ. 25(9), 1567-1580 (2018).

33. Huang D, Zheng X, Wang ZA et al. The MLKL channel in necroptosis is an octamer formed by tetramers in a dyadic process. Mol. Cell. Biol. 37(5), pii: e00497-16 (2017).

34. Callow MG, Watanabe C, Wickliffe KE et al. CRISPR whole-genome screening identifies new necroptosis regulators and RIPK1 alternative splicing. Cell Death Dis. 9(3), 261 (2018). 
35. Xia B, Fang S, Chen X et al. MLKL forms cation channels. Cell. Res. 26(5), 517-528 (2016).

36. Dondelinger Y, Declercq W, Montessuit $S$ et al. MLKL compromises plasma membrane integrity by binding to phosphatidylinositol phosphates. Cell. Rep. 7(4), 971-981 (2014).

- Study revealing the phospholipid-binding and membrane permeabilization by MLKL.

37. Gong YN, Guy C, Olauson H et al. ESCRT-III acts downstream of MLKL to regulate necroptotic cell death and its consequences. Cell 169(2), 286-300; e216 (2017).

- Study revealing ESCRT-III machinery as a general membrane repair antagonist of necroptosis.

38. Ruhl S, Shkarina K, Demarco B, Heilig R, Santos JC, Broz P. ESCRT-dependent membrane repair negatively regulates pyroptosis downstream of GSDMD activation. Science 362(6417), 956-960 (2018).

39. Ros U, Pena-Blanco A, Hanggi $\mathrm{K}$ et al. Necroptosis execution is mediated by plasma membrane nanopores independent of calcium. Cell Rep. 19(1), 175-187 (2017).

40. Conrad M, Angeli JP, Vandenabeele P, Stockwell BR. Regulated necrosis: disease relevance and therapeutic opportunities. Nat. Rev. Drug Discov. 15(5), 348-366 (2016).

41. Rathkey JK, Zhao J, Liu Z et al. Chemical disruption of the pyroptotic pore-forming protein gasdermin D inhibits inflammatory cell death and sepsis. Sci. Immunol. 3(26), (2018).

42. Hildebrand JM, Tanzer MC, Lucet IS et al. Activation of the pseudokinase MLKL unleashes the four-helix bundle domain to induce membrane localization and necroptotic cell death. Proc. Natl Acad. Sci. USA 111(42), 15072-15077 (2014).

43. Ma B, Marcotte D, Paramasivam M et al. ATP-competitive MLKL binders have no functional impact on necroptosis. PLoS One 11(11), e0165983 (2016).

- Study characterizing compounds that bind potently and selectively to the putative nucleotide binding site in human pseudokinase domain, yet that exhibit no activity in necroptosis.

44. Byrne DP, Foulkes DM, Eyers PA. Pseudokinases: update on their functions and evaluation as new drug targets. Future Med. Chem. 9(2), 245-265 (2017).

45. Shears SB, Wang H. Inositol phosphate kinases: expanding the biological significance of the universal core of the protein kinase fold. Adv. Biol. Regul. 71, 118-127 (2019).

46. Frederick JP, Mattiske D, Wofford JA et al. An essential role for an inositol polyphosphate multikinase, Ipk2, in mouse embryogenesis and second messenger production. Proc. Natl Acad. Sci. USA 102(24), 8454-8459 (2005).

47. Wilson MP, Hugge C, Bielinska M, Nicholas P, Majerus PW, Wilson DB. Neural tube defects in mice with reduced levels of inositol 1,3,4-trisphosphate 5/6-kinase. Proc. Natl Acad. Sci. USA 106(24), 9831-9835 (2009).

48. Opferman JT, Letai A, Beard C, Sorcinelli MD, Ong CC, Korsmeyer SJ. Development and maintenance of B and T lymphocytes requires antiapoptotic MCL-1. Nature 426(6967), 671-676 (2003).

49. Kotschy A, Szlavik Z, Murray J et al. The MCL1 inhibitor S63845 is tolerable and effective in diverse cancer models. Nature 538(7626), 477-482 (2016).

50. Green DR, Galluzzi L, Kroemer G. Cell biology. Metabolic control of cell death. Science 345(6203), 1250256 (2014). 